\title{
Hyperosmolar Solutions Effects on Cerebral Oxygenation and Metabolism
}

\author{
S.S. Petrikov ${ }^{*}, 1$, V.V. Krylov ${ }^{1}$, A.A. Solodov ${ }^{1}$, U.V. Titova ${ }^{2}$, H.T. Guseinova ${ }^{2}$, L.T. Khamidova ${ }^{3}$ and \\ A.A. Polupan ${ }^{2}$ \\ ${ }^{I}$ Department of Neurosurgery, ${ }^{2}$ Neurosurgical ICU, ${ }^{3}$ Department of Ultrasound Diagnostics, Sklifosovsky Research \\ Institute, Moscow, Russia
}

\begin{abstract}
Purpose: To estimate the dynamics of cerebral oxygenation and metabolism during intracranial pressure (ICP) correction with $15 \%$ Mannitol and 7,2\% saline in 6\% HES 200/0,5.

Methods: We analyzed 39 episodes of ICP correction with 15\% Mannitol or 7,2\% $\mathrm{NaCl}$ in 6\% HES 200/0,5 ("HyperHAES") in 9 patients with intracranial hemorrhage (GCS 4-8). Monitoring of ICP, systemic hemodynamics, $\mathrm{SvjO}_{2}$ and cerebral microdialysis was used in all patients. Brain temperature (Tbr) and brain oxygen tension $\left(\mathrm{PbrO}_{2}\right)$ were investigated in 5 patients. ICP $>20 \mathrm{mmHg}$ was the indication for treatment.

Results: The duration of ICP reduction below $20 \mathrm{mmHg}$ was $121 \pm 58 \mathrm{~min}$ for $15 \%$ Mannitol and $258 \pm 122 \mathrm{~min}$ for "HyperHAES" $(\mathrm{p}<0,001)$. Administration of the investigated solutions was associated with slight $\mathrm{PbrO}_{2}$ increase. $15 \%$ Mannitol infusion did not change brain metabolism in "intact" and "lesioned" tissue. HyperHAES administration was accompanied with significant increase of glucose and pyruvate concentration in "intact" and "lesioned" brain tissue. We observed the same dynamics of cerebral oxygenation and metabolism in patients with traumatic and nontraumatic intracerebral hemorrhage. Infusion of investigated solutions was not accompanied by significant dynamics of cardiac preload and function.

Conclusions: "HyperHAES" infusion results in prolong ICP reduction than 15\% Mannitol and is accompanied with slight increase of $\mathrm{PbrO}_{2}$ and significant improvement of cerebral metabolism. 15\% Mannitol administration does not influence cerebral oxygenation and metabolism. 15\% Mannitol and "HyperHAES" infusion does not influence systemic hemodynamics in normovolemic patients. Brain lesion, caused by intracranial hemorrhage may be accompanied by mitochondrial dysfunction, characterized by reduction and even enlargement of lactate/pyruvate ratio in condition of sufficient oxygen and glucose delivery to the brain.
\end{abstract}

Keywords: Intracranial hemorrhage, Intracranial pressure, Hyperosmolar solutions, Cerebral oxygenation, Cerebral metabolism.

Brain edema is the main cause of intracranial hypertension in patients with intracranial hemorrhage. In turn, intracranial hypertension aggravates brain edema by altering cerebral perfusion and cerebral venous blood outflow [1-3]. Therefore intracranial pressure (ICP) correction is one of the main goals of intensive care in patients with intracranial hemorrhage. Infusion of hyperosmolar solutions is common therapy of intracranial hypertension [4, 5]. Hyperosmolar solutions increase the osmolar gradient between plasma and cerebral interstitium, and lead to fluid moving from the brain to intravascular space. Infusion of hyperosmolar solutions is accompanied by hypervolemia and reduces blood viscosity, that cause transient increase of cerebral blood flow and reflex vasoconstriction.

The most popular hyperosmolar agents used for intracranial hypertension correction are mannitol (15\% and 20\%) and hypertonic saline $(3 \%, 7.5 \%, 10 \%)$. To increase the duration of their action hyperosmolar solutions are combined with colloids.

*Address correspondence to this author at the Neurosurgical ICU, Sklifosovsky Research Institute, B. Sukharevskaya Sq. 3, 129010 Moscow, Russia; Tel: +79037368669; E-mail: korrida@rambler.ru
In spite of a large amount of investigations, data about hyperosmolar solutions influence on the ICP, systemic hemodynamics and mortality are controversial. There are only few studies, in which effects of mannitol were compared with hypertonic saline [6]. R. Vialet et al., 2003 found that in patients with severe traumatic brain injury (TBI) $7,5 \%$ saline was more effective in intracranial hypertension treatment than 20\% mannitol [7]. C. Battison et al., 2005 investigated the effects of equimolar doses of $20 \%$ mannitol and $7,5 \%$ saline in $6 \%$ dextran-70 in patients with TBI and aneurismal subarachnoid hemorrhage (SAH). Authors mentioned that hypertonic saline combined with colloid solution reduced ICP more effectively than mannitol [8].

Information about effects of hyperosmolar solutions on cerebral oxygenation and metabolism is very small. P.G. Al-Rawi et al., 2005 showed increase of brain oxygen tension and decrease of lactate/pyruvate ratio at $60 \mathrm{~min}$ after reducing the intracranial hypertension with hypertonic saline in patients with poor grade SAH [9]. O.W. Sakowitz et al., 2007 didn't find changes in brain oxygen tension after ICP correction with $20 \%$ mannitol, but showed $10-40 \%$ increase of intracerebral glucose, lactate, pyruvate and glutamate levels in patients with severe TBI [10]. 
Table 1. Patient's Characteristics

\begin{tabular}{|c|c|c|c|c|c|c|c|c|c|c|}
\hline Patient & Sex & Age & Diagnosis & GCS & Surgery & $\begin{array}{l}\text { Second-Tier } \\
\text { Therapy }\end{array}$ & Monitoring & $\begin{array}{l}\text { Number of } \\
15 \% \text { Mannitol } \\
\text { infusions }\end{array}$ & $\begin{array}{c}\text { Number of } \\
\text { "Hyper- } \\
\text { HAES" } \\
\text { Infusions }\end{array}$ & GOS \\
\hline 1 & $\mathrm{~F}$ & 26 & $\begin{array}{c}\text { ASAH } \\
+ \text { Vasospasm }\end{array}$ & 6 & $\begin{array}{l}\text { Aneurism } \\
\text { clipping }\end{array}$ & $\begin{array}{l}\text { Decompressive } \\
\text { craniectomy }\end{array}$ & $\begin{array}{c}\text { ICP } \\
\text { Microdialysis } \\
\text { Licox }\end{array}$ & 4 & 3 & VS \\
\hline 2 & M & 31 & TBI & 7 & $\begin{array}{l}\text { Temporal craniec- } \\
\text { tomy (EDH+IPH) }\end{array}$ & & $\begin{array}{c}\text { ICP } \\
\text { Microdialysis } \\
\text { Licox }\end{array}$ & 4 & 3 & GR \\
\hline 3 & M & 28 & TBI & 7 & $\begin{array}{l}\text { Temporal craniec- } \\
\text { tomy }(\mathrm{SDH})\end{array}$ & & $\begin{array}{c}\text { ICP } \\
\text { Microdialysis }\end{array}$ & 2 & 1 & GR \\
\hline 4 & M & 63 & TBI & 6 & $\begin{array}{l}\text { Temporal craniec- } \\
\text { tomy (SDH+IPH) }\end{array}$ & $\begin{array}{l}\text { Decompressive } \\
\text { craniectomy }\end{array}$ & $\begin{array}{c}\text { ICP } \\
\text { Microdialysis }\end{array}$ & 1 & 1 & $\mathrm{D}$ \\
\hline 5 & M & 31 & TBI & 8 & $\begin{array}{l}\text { Temporal craniec- } \\
\text { tomy (EDH+IPH) }\end{array}$ & & $\begin{array}{c}\text { ICP } \\
\text { Microdialysis } \\
\text { Licox }\end{array}$ & 3 & 2 & GR \\
\hline 6 & $\mathrm{~F}$ & 55 & $\begin{array}{c}\text { ASAH } \\
+ \text { Vasospasm }\end{array}$ & 7 & $\begin{array}{l}\text { Aneurism } \\
\text { clipping }\end{array}$ & $\begin{array}{l}\text { Decompressive } \\
\text { craniectomy }\end{array}$ & $\begin{array}{c}\text { ICP } \\
\text { Microdialysis }\end{array}$ & 1 & 1 & SD \\
\hline 7 & M & 45 & TBI & 5 & $\begin{array}{l}\text { Temporal craniec- } \\
\text { tomy }(\mathrm{SDH})\end{array}$ & $\begin{array}{l}\text { Decompressive } \\
\text { craniectomy }\end{array}$ & $\begin{array}{c}\text { ICP } \\
\text { Microdialysis } \\
\text { Licox }\end{array}$ & 3 & 3 & $\mathrm{D}$ \\
\hline 8 & $\mathrm{~F}$ & 60 & $\begin{array}{c}\text { ASAH } \\
+ \text { Vasospasm }\end{array}$ & 5 & $\begin{array}{l}\text { Aneurism } \\
\text { clipping }\end{array}$ & & $\begin{array}{c}\text { ICP } \\
\text { Microdialysis }\end{array}$ & 1 & 1 & $\mathrm{D}$ \\
\hline 9 & $\mathrm{~F}$ & 69 & $\begin{array}{c}\text { ASAH } \\
+ \text { Vasospasm }\end{array}$ & 6 & $\begin{array}{l}\text { Aneurism } \\
\text { clipping }\end{array}$ & & $\begin{array}{c}\text { ICP } \\
\text { Microdialysis } \\
\text { Licox }\end{array}$ & 3 & 2 & VS \\
\hline
\end{tabular}

M - male; F - female; ASAH - aneurysmal subarachnoid hemorrhage; GCS - Glasgo Coma Score; GOS - Glasgo Outcome Scale 3 months post TBI and SAH ; SDH - subdural hematoma; EDH - epidural hematoma; IPH - intraparenchimal hematoma; ICP - intracranial pressure; GR - good recovery; SD - severe disability; VS - vegetative state; D - dead.

The aim of our study was to estimate the dynamics of cerebral oxygenation and metabolism during intracranial hypertension correction with $15 \%$ mannitol and $7.2 \%$ saline in $6 \%$ HES $200 / 0,5$.

\section{MATERIALS AND METHODOLOGY}

Investigation was approved by local ethical committee.

We analyzed 39 episodes of increased ICP correction with hyperosmolar solutions in 9 patients with intracranial hemorrhage and Glasgow Coma Score 4-8. Four patients were with aneurismal SAH and 5 patients - with severe TBI (Table 1). All patients were operated on. Mean age was $45 \pm$ 17 years, male/female ratio - 5/4.

In all patients with SAH the linear blood flow velocity in middle brain arteries (MBA) was determined by transcranial dopplerography ("MultiDop T", DWL Elektronische Systeme $\mathrm{GmbH}$, Germany). Severe vasospasm (blod flow velocity in the left and right MBA more than $200 \mathrm{~cm} / \mathrm{sec}$ ) was observed in all patients.

Monitoring of ICP, systemic hemodynamics, oxygen saturation in the internal jugular vein bulb $\left(\mathrm{SvjO}_{2}\right)$ and cerebral microdialysis was used in all patients. Brain temperature (Tbr) and brain oxygen tension $\left(\mathrm{PbrO}_{2}\right)$ were investigated in five patients.

\section{ICP Monitoring}

The special double-lumen catheter with a balloon at the edge («Air-Pouch Probe, 3XL», Germany) was placed in anterior horn of right or left lateral ventricle for intraventricular ICP measurement. Measuring lumen of catheter was connected to the monitoring system «Spiegelberg: BrainPressure Monitor» (Germany), draining lumen - to the CSF drainage system.

For intraparenchymatous ICP measurement «Codman MicroSensor ${ }^{\mathrm{MM}} \gg$ probe (USA) was used. Probes were placed in the frontal or temporal lobe of unlesioned brain hemisphere and connected to the «Codman ICP Express» monitor (USA).

\section{Hemodynamic Monitoring}

Systemic hemodynamics was estimated by transpulmonary thermodilution («PiCCOplus», Pulsion Medical Systems, Germany). One of the subclavian or internal jugular veins was catheterized and special thermistor-tipped catheter (Pulsiocath PV2015L20 «Pulsion Medical Systems», Germany) was placed in femoral artery. Arterial access allowed continuous monitoring of hemodynamic values and arterial blood sampling. Transpulmonary thermodilution was performed at all stages of the study (Table 2). Arterial blood pressure transducer was fixed on the level of foramen Monro (the middle of the length between external orbit angle and external acoustic meatus).

\section{Jugular Oxymetry}

For $\mathrm{SvjO}_{2}$ measurement central venous catheter was placed into bulb of jugular vein. The position of catheter was verified via side view X-ray of cervix. Venous blood probes 
were analyzed in the laboratory (Gas analyzer "ABL 800", Radiometr, Denmark).

Table 2. Basic Parameters of Systemic Hemodynamics, Measured by Transpulmonary Thermodilution

\begin{tabular}{|l|c|}
\hline \multicolumn{1}{|c|}{ Parameter } & Normal Values \\
\hline \hline Cardiac index $\left(1 / \mathrm{min} / \mathrm{m}^{2}\right)$ & $3-5$ \\
\hline Global end-diastolic volume index $\left(\mathrm{ml} / \mathrm{m}^{2}\right)$ & $680-800$ \\
\hline System vascular resistance index $\left(\mathrm{dyn} * \mathrm{sec}^{*} \mathrm{~cm}^{-5} / \mathrm{m}^{2}\right)$ & $1200-2000$ \\
\hline Extravascular lung water index $(\mathrm{ml} / \mathrm{kg})$ & $3-7$ \\
\hline Stroke volume variation $(\%)$ & $<10$ \\
\hline Cardiac function index $(1 / \mathrm{min})$ & $4,5-6,5$ \\
\hline
\end{tabular}

\section{Cerebral Microdialysis}

Cerebral microdialysis catheters (CMA 70, membrane length $10 \mathrm{~mm}$, pore diameter $20000 \mathrm{Da}$, Sweden) were inserted through the drill hole or special fixation device ("bolt") into "lesioned" (in patients with TBI - brain tissue near primary lesion, in patients with ASAH - brain tissue perfused by involved artery) and "intact" brain tissue (Fig. 1). Catheters location was confirmed by X-ray computer tomography (CT).
Catheter was perfused with CNS Perfusion Fluid (CMA Microdialysis, Sweden) at rate $0,3 \mu \mathrm{L} / \mathrm{min}$ using CMA 107 perfusion pumps (CMA Microdialysis, Sweden) after the insertion. It took approximately $17 \mathrm{~min}$ to accumulate enough volume of dialysate. Vials with dialysate were placed into «ISCUS Clinical Microdialysis Analyser» (CMA Microdialysis, Sweden) to measure glucose, glycerol, lactate and piruvate concentrations (Table $\mathbf{3}$ ).

\section{Monitoring of Brain Oxygen Pressure and Brain Tem- perature}

Polarographic $\mathrm{PbrO}_{2}$ electrodes ( REVOXODE Brain Oxygen Catheter-Micro-Probe», Integra Lifesciences, Germany) and brain temperature probes («TRERMOCOUPLE Brain Temperature Catheter-Micro-Probe», Integra Lifesciences, Germany) were placed into "lesioned" and "intact" brain tissue through special fixation device ("bolt"). Probes location in the brain was confirmed by CT. Probes were connected to «LICOX ${ }_{C M P}{ }^{\circledR}$ Tissue Oxygen Pressure Monitor» (Integra Lifesciences, Germany).

\section{Patient Management}

Standard intensive care was used for all patients. Infusion therapy was based on combination of colloid and crystalloid solutions. Decision about infusion therapy volume and structure was based on invasive homodynamic monitoring data.
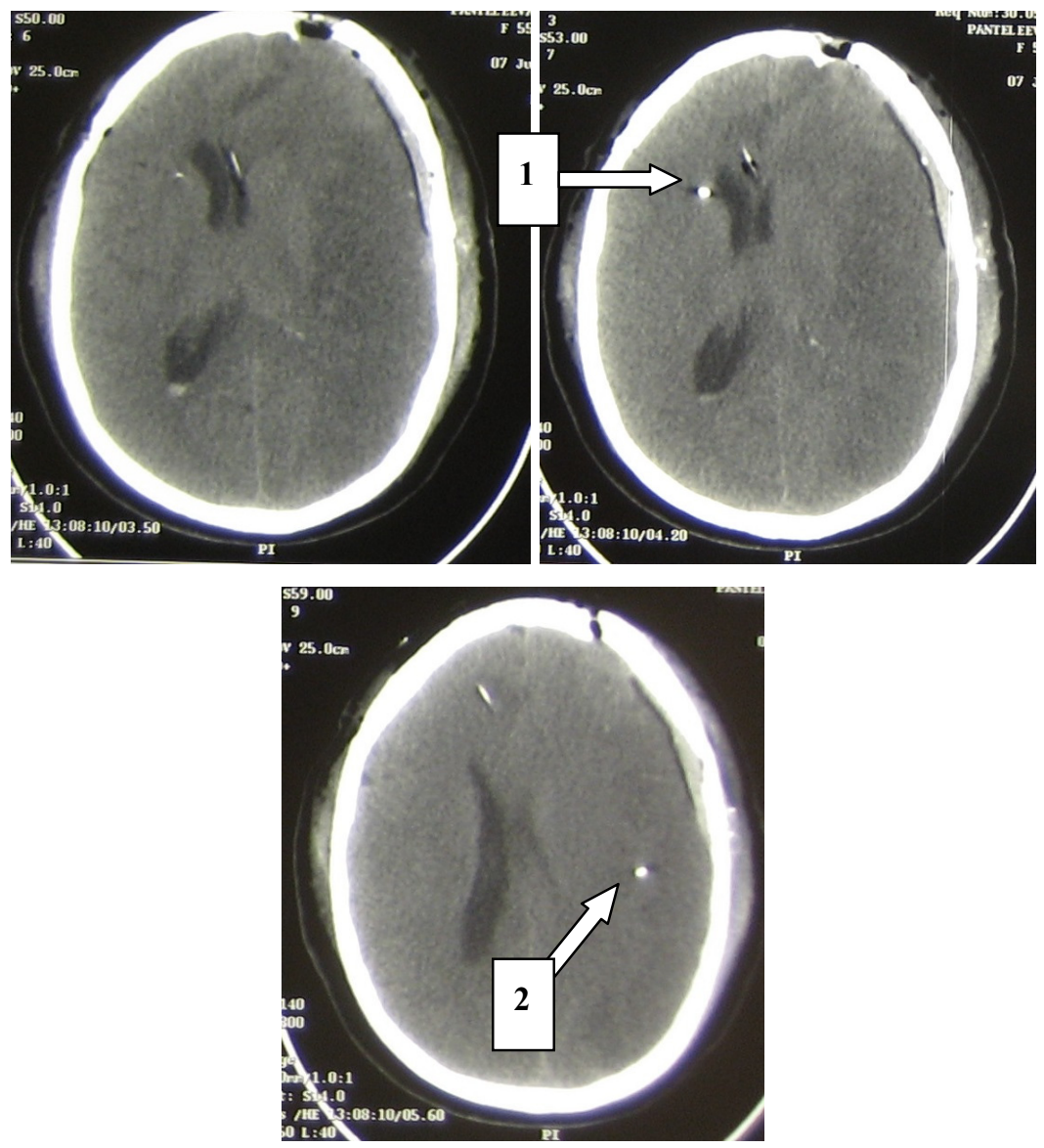

Fig. (1). CT scan of the patient with vasospasm after left internal carotid artery aneurysm rupture. Microdialysis catheters are located in "intact" (1) and "lesioned" (2) brain tissue. 
Enteral feeding (20-25 kkal/ $\mathrm{kg} /$ day) was started on the first day after the admission to the ICU. Nitrogen balance was calculated for protein requirement evaluation. Parenteral nutrition was added if necessary. Artificial lung ventilation with tidal volume $8-10 \mathrm{ml} / \mathrm{kg}$ of ideal body mass and PEEP 5 $\mathrm{cmH}_{2} \mathrm{O}$ was administered in all cases. Target $\mathrm{PaCO}_{2}$ level was $30-40 \mathrm{mmHg}$. Head-edge of the bed was elevated to $30-40^{\circ}$. Analgetic and sedative drugs (propofol infusion (3 - $6 \mathrm{mg} / \mathrm{kg} /$ hour) or bolus dosing of midazolam $(0,15-0,25$ $\mathrm{mg} / \mathrm{kg})$ with nalbufine $200-300 \quad(\mathrm{mkg} / \mathrm{kg}))$ were used during invasive procedures (tracheotomy, vessel catheterizations), to achieve target $\mathrm{PaCO}_{2}$ level and in patients with psychomotor agitation.

Table 3. Metabolites, Measured by Cerebral Microdialysis [11]

\begin{tabular}{|l|c|}
\hline \multicolumn{1}{|c|}{ Parameters } & Normal Values \\
\hline \hline Glucose $(\mathrm{mmol} / \mathrm{l})$ & $1,7 \pm 0,9$ \\
\hline Pyruvate $(\mu \mathrm{cmol} / \mathrm{l})$ & $166 \pm 47$ \\
\hline Lactate $(\mathrm{mmol} / \mathrm{l})$ & $2,9 \pm 0,9$ \\
\hline Lactate $/$ pyruvate ratio & $23 \pm 4$ \\
\hline Glycerol $(\mu \mathrm{cmol} / \mathrm{l})$ & $80 \pm 40$ \\
\hline
\end{tabular}

\section{Study Design}

Persistent elevation (during 15-20 minutes) of intracranial pressure above $20 \mathrm{mmHg}$, tolerant to routine methods of ICP correction (elevation of the bed head-edge, maintenance of normothermia and normoxia, $\mathrm{PaCO}_{2}$ 30-35 $\mathrm{mmHg}$, analgesia and sedation) was the indication for hyperosmolar solutions infusion.

Intravenous infusion of $15 \%$ Mannitol (Biochimik, Russian Federation), $400 \mathrm{ml}(0,5-1 \mathrm{~g} / \mathrm{kg})$ during 15-20 min $(\mathrm{n}=22)$ or "HyperHAES" $(7,2 \% \mathrm{NaCl}$ in $6 \%$ HES $200 / 0,5)$ (Fresenius Kabi Deutschland GmbH, Germany), $250 \mathrm{ml}$
(2 - $4 \mathrm{ml} / \mathrm{kg})$ during 5-15 $\min (\mathrm{n}=17)$ were used for ICP reduction in accordance with intrahospital protocol.

Each patient received infusion of both solutions. For example - first medication was 15\% Mannitol, second "HyperHAES", third - 15\% Mannitol etc. Patients were randomized to receive 15\% Mannitol or "HyperHAES" first. In 5 patients first medication was 15\% Mannitol first, in four "HyperHAES". Minimal interval between infusions of different solutions was $120 \mathrm{~min}$.

The arterial hemoglobin concentration $(\mathrm{Hb}), \mathrm{ICP}$, mean arterial blood pressure (MAP), heart rate (HR), arterial blood temperature (Tbl), Tbr, $\mathrm{PbrO}_{2}$, oxygen extraction ratio $\left(\mathrm{O}_{2}\right.$ ER), cardiac index $(\mathrm{CI})$, cardiac function index $(\mathrm{CFI})$, global end-diastolic volume index (GEDVI), stroke volume variation (SVV), systemic vascular resistance index (SVRI), cerebral perfusion pressure (CPP), $\mathrm{SvjO}_{2}, \mathrm{PaO}_{2}, \mathrm{PaCO}_{2}$, $\mathrm{PaO}_{2} / \mathrm{FiO}_{2}$, serum glucose concentration, glucose, lactate, pyruvate, glycerol concentrations and lactate/pyruvate ratio in cerebral interstitial fluid were measured before infusion and 30 and $120 \mathrm{~min}$ after it. Metabolites measured by cerebral microdialysis, Tbr and $\mathrm{PbrO}_{2}$ were analyzed in "intact" (int) and "lesioned" (les) brain tissue.

\section{Statistical Analysis}

All the parameters investigated were compared between the groups ("Mannitol" vs "HyperHAES") and inside each group. Distribution normality was evaluated by Kolmogorov-Smirnov's criterion. Intragroup differences were evaluated with Wilcoxon's signed-rank test. Intergroup differences were evaluated with Mann-Whitney test. For all analyses $\mathrm{p}$ value of $<0,05$ was regarded as significant. Data are expressed as $\mathrm{M} \pm \mathrm{SD}$ (M -mean, $\mathrm{SD}$ - standard deviation) if distribution was normal and as median (25 and 70 percentile) if distribution was abnormal.

\section{RESULTS}

$\mathrm{FiO}_{2}, \mathrm{Hb}, \mathrm{PaO}_{2}, \mathrm{PaCO}_{2}, \mathrm{PaO}_{2} / \mathrm{FiO}_{2}, \mathrm{Tbl}$ and serum glucose concentration were stable and comparable between groups during the study (Table 4).

Table 4. $\mathrm{FiO}_{2}, \mathrm{Hb}(\mathrm{g} / \mathrm{l}), \mathrm{PaCO}_{2}(\mathrm{mmHg}), \mathrm{PaO}_{2}(\mathrm{mmHg}), \mathrm{PaO}_{2} / \mathrm{FiO}_{2}$, Tbl $\left({ }^{\circ} \mathrm{C}\right)$ and Serum Glucose Concentration (mmol/l) During the Study

\begin{tabular}{|c|c|c|c|c|c|c|c|}
\hline \multirow{2}{*}{ Solutions } & \multicolumn{7}{|c|}{ Study Stages } \\
\hline & $\mathrm{FiO}_{2}$ & $\mathbf{H b}$ & $\mathrm{PaCO}_{2}$ & $\mathrm{PaO}_{2}$ & $\mathrm{PaO}_{2} / \mathrm{FiO}_{2}$ & Tbl & Glu \\
\hline 15\% Mannitol & $0,5 \pm 0,1$ & $88 \pm 17$ & $31 \pm 4$ & $160 \pm 42$ & $310 \pm 92$ & $37,6 \pm 0,7$ & $6,9 \pm 1,9$ \\
\hline HyperHAES & $0,5 \pm 0,1$ & $82 \pm 18$ & $30 \pm 4$ & $184 \pm 65$ & $348 \pm 78$ & $37,6 \pm 0,5$ & $7,5 \pm 2,7$ \\
\hline $15 \%$ Mannitol & $0,5 \pm 0,1$ & $94 \pm 24$ & $33 \pm 4$ & $157 \pm 42$ & $303 \pm 102$ & $37,4 \pm 0,7$ & $6,6 \pm 1,6$ \\
\hline \multirow[t]{2}{*}{ HyperHAES } & $0,5 \pm 0,1$ & $83 \pm 19$ & $31 \pm 5$ & $166 \pm 35$ & $335 \pm 78$ & $37,4 \pm 0,8$ & $7,3 \pm 2,9$ \\
\hline & \multicolumn{7}{|c|}{120 Min After Infusion } \\
\hline
\end{tabular}




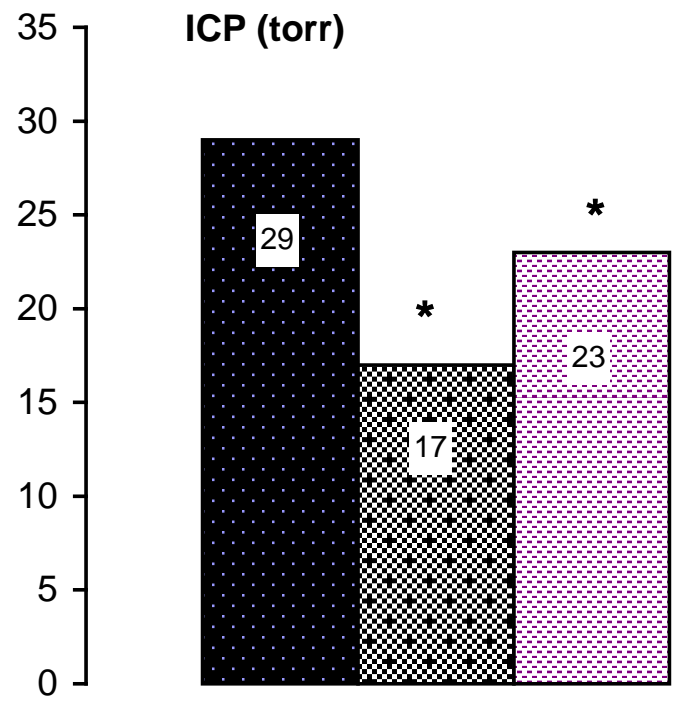

15\% Mannitol

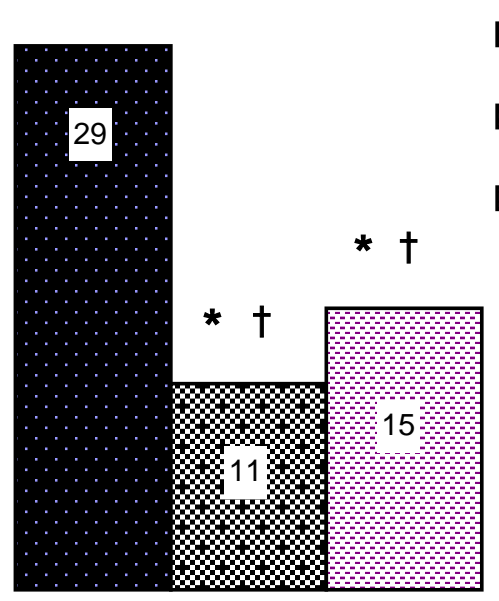

HyperHAES
Initial

国30 $\mathrm{min}$

圄 $120 \min$

Fig. (2). ICP dynamics after 15\% Mannitol ( $\mathrm{n}=22)$ and "HyperHAES" ( $\mathrm{n}=17)$ infusion. * ${ }^{*} \mathrm{p}<0,05$ vs initial values, ${ }^{\dagger}-\mathrm{p}<0,05 v s 15 \%$ Mannitol.

Table 5. Effects of Hyperosmolar Solutions on the ICP (mmHg) and CPP (mmHg)

\begin{tabular}{|c|c|c|c|c|c|c|}
\hline \multirow{2}{*}{ Solutions } & \multicolumn{6}{|c|}{ Study Stages } \\
\hline & ICP & CPP & ICP & CPP & ICP & CPP \\
\hline 15\% Mannitol & $29 \pm 5$ & $73 \pm 14$ & $17 \pm 5^{*}$ & $89 \pm 16^{*}$ & $23 \pm 9^{*}$ & $81 \pm 13$ \\
\hline HyperHAES & $29 \pm 5$ & $71 \pm 14$ & $11 \pm 5^{*, \dagger}$ & $93 \pm 11 *$ & $15 \pm 6^{*, \dagger}$ & $87 \pm 15^{*}$ \\
\hline
\end{tabular}

${ }^{*}$ - $\mathrm{p}<0,05 v s$ initial values, ${ }^{\dagger}$ - $\mathrm{p}<0,05 v s 15 \%$ Mannitol.

\section{Intracranial and Cerebral Perfusion Pressures}

Mean ICP was $29 \pm 5 \mathrm{mmHg}$ before $15 \%$ Mannitol and "HyperHAES" infusions. Introduction of investigated solutions significantly decreased ICP at 30 and $120 \mathrm{~min}$ after infusion (Table 5). But "HyperHAES" had more pronounced effect on ICP than mannitol (Fig. 2).
The duration of ICP reduction below $20 \mathrm{mmHg}$ was 121 \pm 58 min for $15 \%$ Mannitol and $258 \pm 122 \mathrm{~min}$ for "HyperHAES" $(\mathrm{p}<0,001)$.

$15 \%$ Mannitol administration was accompanied by significant increase of cerebral perfusion pressure at $30 \mathrm{~min}$ after infusion (Table 5). "HyperHAES" introduction increased significantly CPP at 30 and 120 min after infusion.

Table 6. Hyperosmolar Solutions Effects on Systemic Hemodynamics

\begin{tabular}{|c|c|c|c|c|c|c|}
\hline \multirow{3}{*}{ Parameters } & \multicolumn{6}{|c|}{ Study Stages } \\
\hline & \multicolumn{2}{|c|}{ Before Infusion } & \multicolumn{2}{|c|}{30 Min After Infusion } & \multicolumn{2}{|c|}{120 Min After Infusion } \\
\hline & 15\% Mannitol & HyperHAES & $15 \%$ Mannitol & HyperHAES & 15\% Mannitol & HyperHAES \\
\hline $\mathrm{CI}, \mathrm{l} / \mathrm{min} / \mathrm{m}^{2}$ & $4,5 \pm 0,8$ & $4,6 \pm 1,0$ & $4,9 \pm 1,0$ & $5,0 \pm 1,1^{*}$ & $4,7 \pm 0,9$ & $4,7 \pm 0,9$ \\
\hline GEDVI, $\mathbf{m l} / \mathbf{m}^{2}$ & $779 \pm 152$ & $740 \pm 70$ & $767 \pm 141$ & $742 \pm 96$ & $767 \pm 202$ & $758 \pm 101$ \\
\hline SVV, \% & $13 \pm 5$ & $13 \pm 6$ & $11 \pm 4$ & $10 \pm 4$ & $15 \pm 4$ & $13 \pm 5$ \\
\hline MAP, mmHg & $102 \pm 14$ & $99 \pm 14$ & $106 \pm 16$ & $104 \pm 11$ & $104 \pm 12$ & $102 \pm 13$ \\
\hline HR beat/min & $75 \pm 17$ & $76 \pm 13$ & $82 \pm 16^{*}$ & $79 \pm 13$ & $83 \pm 17^{*}$ & $76 \pm 13$ \\
\hline SVRI, dyn* $\sec ^{*} \mathrm{~cm}^{-5} / \mathrm{m}^{2}$ & $1724 \pm 460$ & $1760 \pm 460$ & $1738 \pm 377$ & $1633 \pm 411$ & $1658 \pm 359$ & $1768 \pm 498$ \\
\hline $\mathrm{CFI}, \mathbf{l} / \mathrm{min}$ & $5,9 \pm 1,2$ & $6,2 \pm 1,4$ & $6,4 \pm 1,1$ & $6,7 \pm 1,3$ & $6,3 \pm 1,1$ & $6,2 \pm 1,4$ \\
\hline
\end{tabular}

${ }^{*}$ - $\mathrm{p}<0,05$ vs initial values. 
Table 7. Cerebral Oxygenation and Metabolism in Patients with Traumatic Brain Injury and Aneurismal Subarachnoid Hemorrhage Before Investigation

\begin{tabular}{|c|c|c|c|c|c|c|c|c|}
\hline Diagnosis & $\begin{array}{c}\mathrm{PbrO}_{2}(\mathrm{int}) \\
(\mathrm{mmHg})\end{array}$ & $\begin{array}{c}\mathrm{PbrO}_{2}(\mathrm{les}) \\
\text { (mmHg) }\end{array}$ & $\begin{array}{c}\text { Glucose(int) } \\
(\mathrm{mmol} / \mathrm{l})\end{array}$ & $\begin{array}{c}\text { Glucose(les) } \\
(\mathrm{mmol} / \mathrm{l})\end{array}$ & $\mathbf{L} / \mathbf{P}$ (int) & L/P (les) & $\begin{array}{c}\text { Glycerol } \\
\text { (int) } \mu \mathrm{cmol} / \mathrm{l}\end{array}$ & $\begin{array}{c}\text { Glycerol (les) } \\
\mu \mathrm{cmol} / \mathrm{l}\end{array}$ \\
\hline TBI & $\begin{array}{c}38,3 \pm 16,5 \\
(\mathrm{n}=4)\end{array}$ & $\begin{array}{c}29,1 \pm 14,7 \\
(\mathrm{n}=4)\end{array}$ & $\begin{array}{c}0,9 \pm 0,3 \\
(\mathrm{n}=5)\end{array}$ & $\begin{array}{c}0,8 \pm 0,2 \\
(\mathrm{n}=5)\end{array}$ & $\begin{array}{c}34,4 \pm 10,7 \\
(\mathrm{n}=5)\end{array}$ & $\begin{array}{c}30,8 \pm 8,7 \\
(\mathrm{n}=5)\end{array}$ & $\begin{array}{c}87,4 \pm 42,7 \\
(\mathrm{n}=5)\end{array}$ & $\begin{array}{c}195(147 ; 213) \\
(\mathrm{n}=5)\end{array}$ \\
\hline ASAH & $\begin{array}{l}31,7 \pm 1,7 \\
\quad(\mathrm{n}=2)\end{array}$ & $\begin{array}{c}26,9 \pm 13,2 \\
(\mathrm{n}=2)\end{array}$ & $\begin{array}{c}1,2 \pm 0,6 \\
(\mathrm{n}=4)\end{array}$ & $\begin{array}{c}0,7 \pm 0,3 \\
(\mathrm{n}=4)\end{array}$ & $\begin{array}{l}22,1 \pm 6,3 \\
\quad(n=4)\end{array}$ & $\begin{array}{c}50,3(41 ; 102) \\
(\mathrm{n}=4)\end{array}$ & $\begin{array}{c}68 \pm 46,5 \\
(\mathrm{n}=4)\end{array}$ & $\begin{array}{c}102(47 ; 359) \\
(\mathrm{n}=4)\end{array}$ \\
\hline
\end{tabular}

TBI - traumatic brain injury; ASAH - aneurismal subarachnoid hemorrhage; $n$ - number of patients.

\section{Systemic Hemodynamics Parameters}

Patients in both groups were normovolemic before infusion of the investigated solutions (Table 6). $15 \%$ Mannitol infusion was accompanied by non-significant influence on systemic hemodynamics. "HyperHAES" administration increased significantly cardiac output and decreased slightly SVV at $30 \mathrm{~min}$ after infusion. Infusion of investigated solutions was not accompanied by significant dynamics of global end-diastolic volume and cardiac function.

\section{Cerebral Oxygenation and Metabolism}

Brain oxygen tension and cerebral metabolites levels were comparable between patients with TBI and ASAH before the investigation (Table 7). During investigation we observed the same dynamics of cerebral oxygenation and metabolism in patients with traumatic and nontraumatic intracerebral hemorrhage.

All studied episodes of intracranial hypertension were not accompanied by $\mathrm{PbrO}_{2}$ reduction below ischemic threshold $\left(15 \mathrm{mmHg}\right.$ ) and significant increase of $\mathrm{O}_{2}$ ER (Table 8, Figs. $3,4)$. Brain oxygen pressure was within normal values and was slightly higher in "intact" tissue.

Administration of the investigated solutions was associated with slight $\mathrm{PbrO}_{2}$ increase in "intact" and "lesioned" brain tissue. However, "HyperHAES" administration significantly increased $\mathrm{PbrO}_{2}$ (les) at 30min following infusion (Fig. 4).

Brain temperature in "intact" and "lesioned" tissue was equal and did not change during the study. $15 \% \quad$ Mannitol and "HyperHAES" infusion did not influence oxygen extraction ratio.

Reduction of glucose and pyruvate and slight increase of glycerol concentration in "lesioned" and "intact" brain tissue was observed initially in all patients (Table 8, Figs. 5-8). Lactate concentration was within normal values or nonsignificantly exceeded them. Lactate/pyruvate ratio exceeded normal values in "intact" and "lesioned" brain tissue.

15\% Mannitol infusion did not change glucose, pyruvate concentrations and lactate/pyruvate ratio in "intact" and "lesioned" tissue.

HyperHAES administration was accompanied with significant increase of glucose concentration in "intact" and "lesioned" brain tissue (Figs. 5, 6). Glucose concentration in "lesioned" tissue at $120 \mathrm{~min}$ after "HyperHAES" infusion was significantly higher in comparison with $15 \%$ Mannitol $(1,25 \quad(0,8-1,4) \quad \mathrm{mmol} / 1$ and $0,7 \quad(0,5-1,0), \quad \mathrm{mmol} / 1$ respectively) (Fig. 6).

Pyruvate concentration increased in "intact" and in "lesioned" brain tissue as well (Figs. 7, 8). Lactate concentration significantly increased in "intact" and

Table 8. Hyperosmolar Solutions Effects on $\mathrm{O}_{2} \mathrm{ER}$ and Microdialysis Lactate, Glycerol and Lactate/Pyruvate Ratio

\begin{tabular}{|c|c|c|c|c|c|c|}
\hline \multirow{3}{*}{ Parameters } & \multicolumn{6}{|c|}{ Study Stages } \\
\hline & \multicolumn{2}{|c|}{ Before Infusion } & \multicolumn{2}{|c|}{30 Min After Infusion } & \multicolumn{2}{|c|}{120 Min After Infusion } \\
\hline & 15\% Mannitol & HyperHAES & 15\% Mannitol & HyperHAES & 15\% Mannitol & HyperHAES \\
\hline $\mathrm{O}_{2} \mathrm{ER}$ & $0,23 \pm 0,09$ & $0,21 \pm 0,1$ & $0,19 \pm 0,07$ & $0,19 \pm 0,06$ & $0,19 \pm 0,08$ & $0,21 \pm 0,08$ \\
\hline $\begin{array}{l}\text { Lactate (int), } \\
\mathrm{mmol} / 1\end{array}$ & $\begin{array}{c}2,5 \\
(1,5-3,8)(\mathrm{n}=22)\end{array}$ & $\begin{array}{c}2,2 \\
(1,5-2,6)(\mathrm{n}=17)\end{array}$ & $\begin{array}{c}2,7 * \\
(2,0-4,9)\end{array}$ & $\begin{array}{c}3,0^{*} \\
(2,7-3,9)\end{array}$ & $\begin{array}{c}2,8 \\
(1,9-3,3)\end{array}$ & $\begin{array}{c}3,3 * \\
(2,5-4,8)\end{array}$ \\
\hline $\begin{array}{l}\text { Lactate (les), } \\
\mathrm{mmol} / 1\end{array}$ & $\begin{array}{c}3,2 \\
(1,4-4,5)(n=22)\end{array}$ & $\begin{array}{c}2,7 \\
(1,7-4,0)(n=17)\end{array}$ & $\begin{array}{c}4,1^{*} \\
(1,7-5,5)\end{array}$ & $\begin{array}{c}3,0^{*} \\
(2,4-5,2)\end{array}$ & $\begin{array}{c}4,0^{*} \\
(2,0-5,6)\end{array}$ & $\begin{array}{c}2,95^{*} \\
(2,5-5,1)\end{array}$ \\
\hline Lactate/Pyruvate (int) & $\begin{array}{c}29,8 \\
(24,8-32,7)(n=22)\end{array}$ & $\begin{array}{c}27 \\
(22-30,7)(\mathrm{n}=17)\end{array}$ & $\begin{array}{c}26,8 \\
(24,3-32,6)\end{array}$ & $\begin{array}{c}28,3 \\
(26,1-31,7)\end{array}$ & $\begin{array}{c}27,4 \\
(25,5-31,8)\end{array}$ & $\begin{array}{c}31,9 * \\
(25,9-38,7)\end{array}$ \\
\hline Lactate/Pyruvate (les) & $\begin{array}{c}29,2 \\
(25,9-37,4)(\mathrm{n}=22)\end{array}$ & $\begin{array}{c}29,9 \\
(26,4-35,3)(\mathrm{n}=17)\end{array}$ & $\begin{array}{c}30,3 \\
(25,8-38,5)\end{array}$ & $\begin{array}{c}30,5 \\
(25,9-34,1)\end{array}$ & $\begin{array}{c}31 \\
(25,8-38,3)\end{array}$ & $\begin{array}{c}31,3 \\
(26,7-32,9)\end{array}$ \\
\hline $\begin{array}{c}\text { Glycerol (int) } \\
\mu \mathrm{cmol} / 1\end{array}$ & $\begin{array}{c}89 \\
(66-133)(n=22)\end{array}$ & $\begin{array}{c}107 \\
(59-167)(\mathrm{n}=17)\end{array}$ & $\begin{array}{c}95 \\
(41-153)\end{array}$ & $\begin{array}{c}132 \\
(60-201)\end{array}$ & $\begin{array}{c}94 \\
(76-132)\end{array}$ & $\begin{array}{c}121 \\
(81-185)\end{array}$ \\
\hline $\begin{array}{l}\text { Glycerol (les), } \\
\mu \mathrm{cmol} / 1\end{array}$ & $\begin{array}{c}59 \\
(49-136)(n=22)\end{array}$ & $\begin{array}{c}91 \\
(46-245)(n=17)\end{array}$ & $\begin{array}{c}65 \\
(30-120)\end{array}$ & $\begin{array}{c}95 \\
(61-263)\end{array}$ & $\begin{array}{c}74 \\
(61,5-158)\end{array}$ & $\begin{array}{c}115 \\
(83-178)\end{array}$ \\
\hline
\end{tabular}




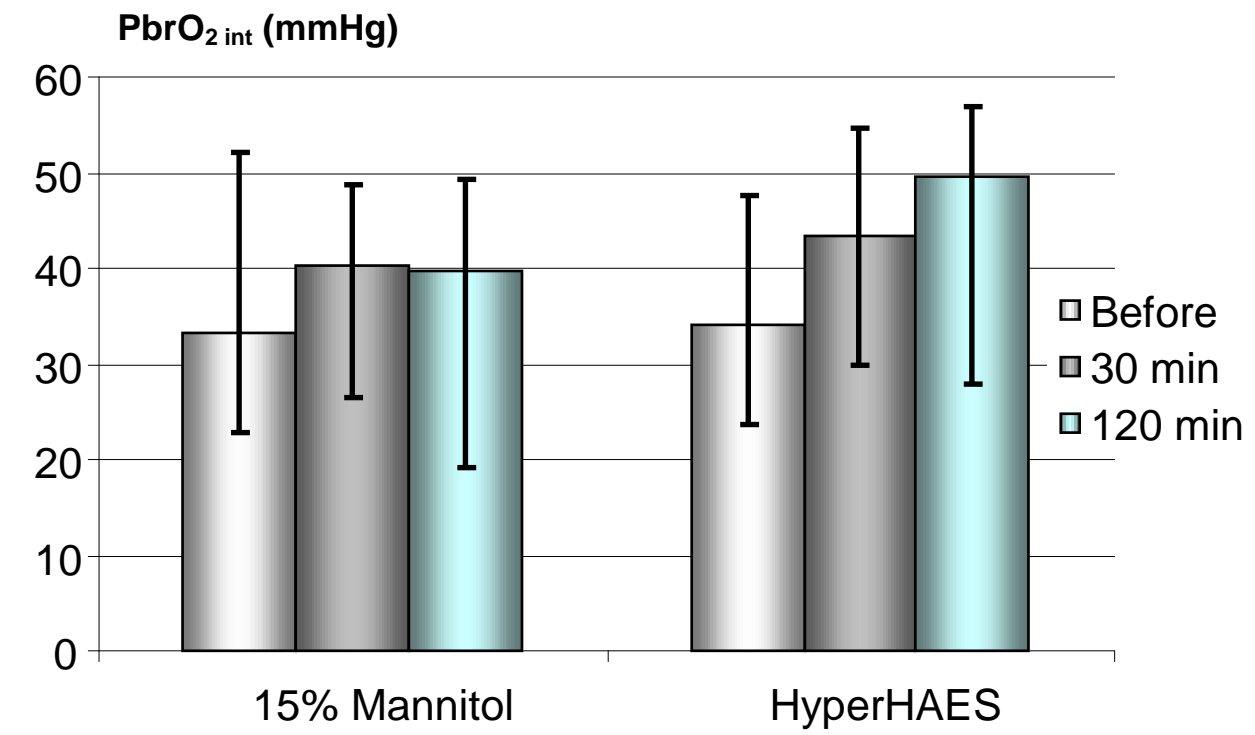

Fig. (3). 15\% Mannitol ( $\mathrm{n}=17)$ and "HyperHAES" ( $\mathrm{n}=13)$ effects on $\mathrm{PbrO}_{2}$ (int) (columns - median; errors: lower - 25 percentile, upper - 75 percentile).

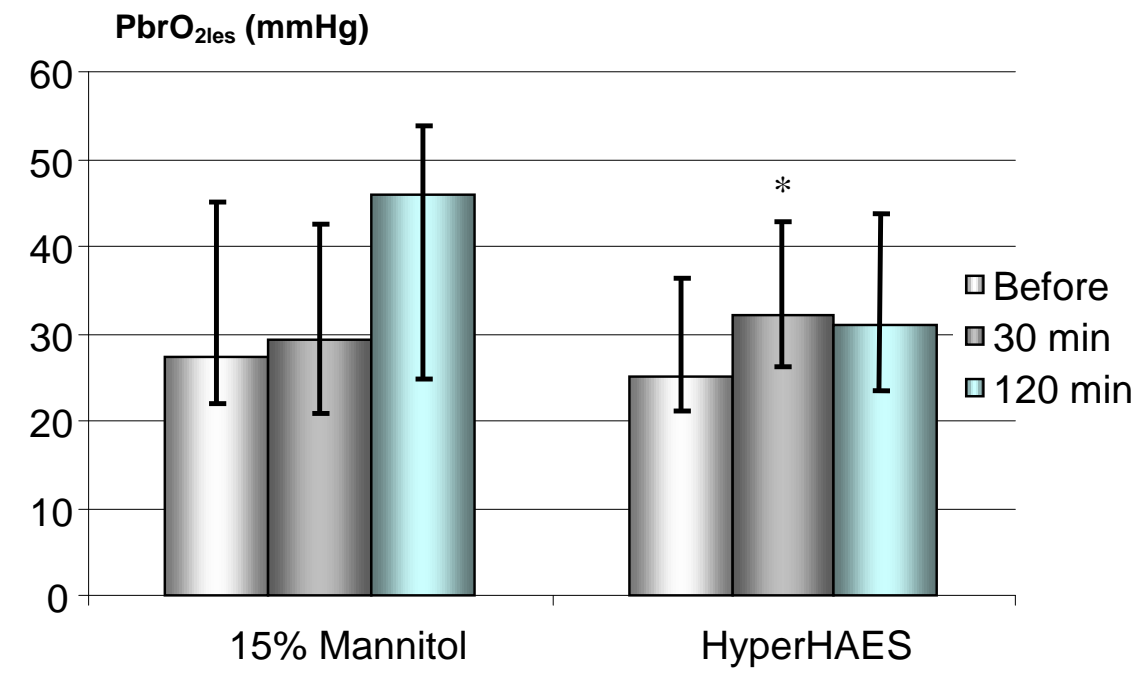

Fig. (4). 15\% Mannitol (n=17) and "HyperHAES" (n=13) effects on $\mathrm{PbrO}_{2}$ les (columns - median; errors: lower - 25 percentile, upper - 75 percentile); $*-\mathrm{p}<0,05$ vs initial values.

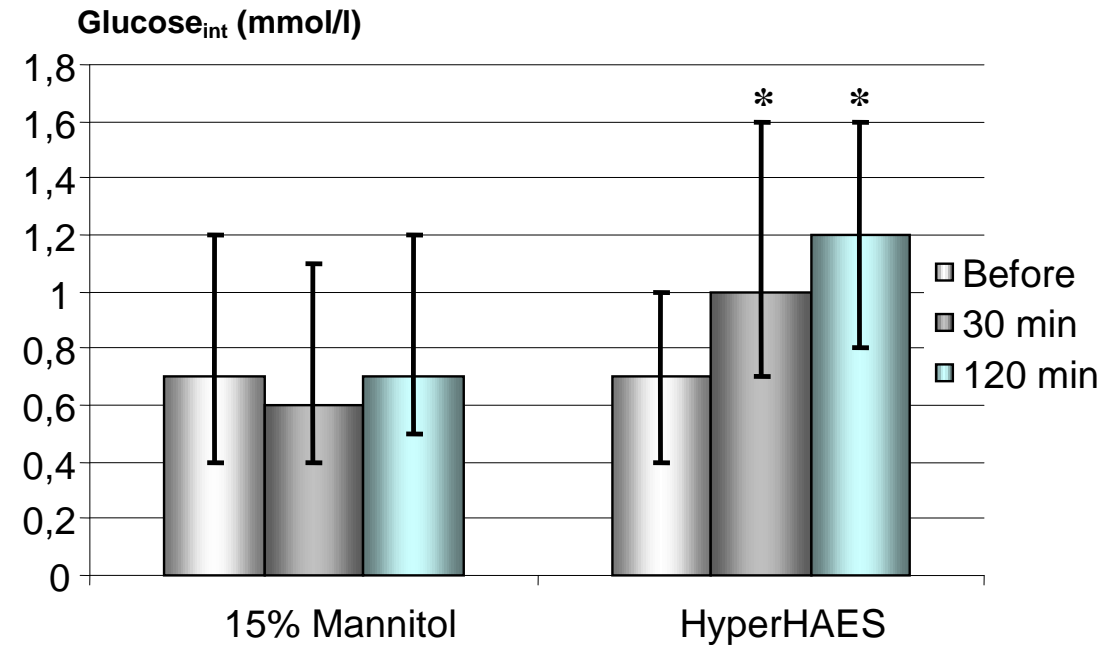

Fig. (5). 15\% Mannitol (n=22) and "HyperHAES" ( $n=17)$ effects on glucose concentration in "intact" brain tissue (columns - median; errors: lower - 25 percentile, upper - 75 percentile); * ${ }^{*}<0,05$ vs initial values. 


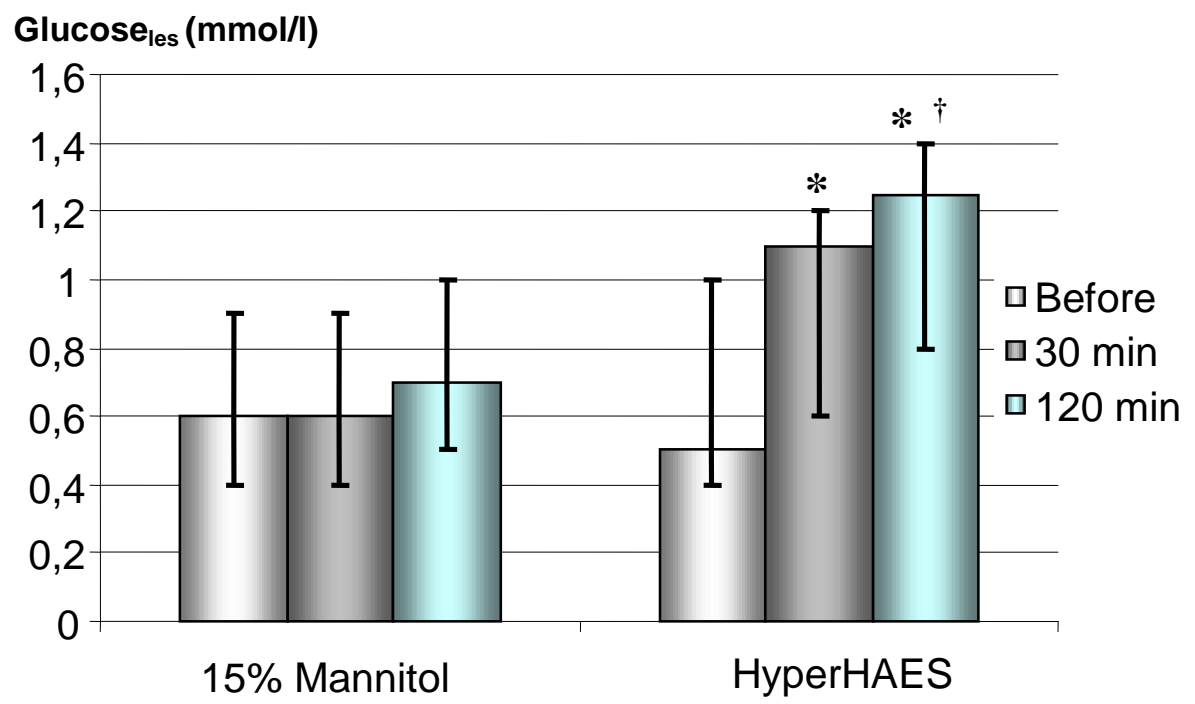

Fig. (6). 15\% Mannitol ( $\mathrm{n}=22)$ and "HyperHAES" $(\mathrm{n}=17)$ effects on glucose concentration in "lesioned" brain tissue (columns - median; errors: lower - 25 percentile, upper - 75 percentile); * ${ }^{*}<0,05$ s initial values, ${ }^{\dagger}$ - $\mathrm{p}<0,05$ vs $15 \%$ Mannitol.

\section{Pyruvate $_{\text {int }}(\mu \mathrm{cmol} / \mathrm{l})$}

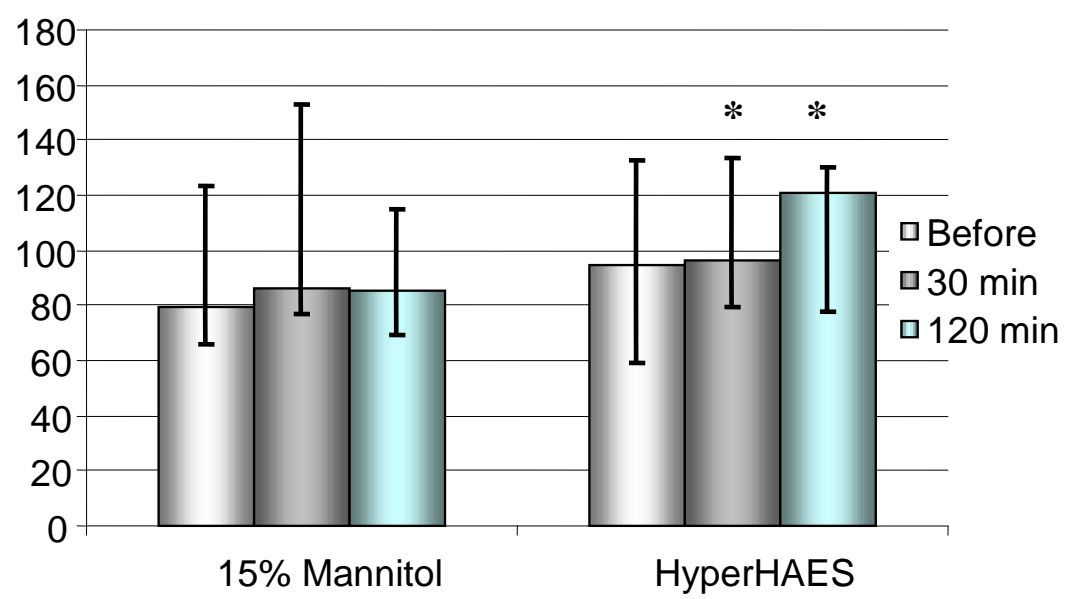

Fig. (7). 15\% Mannitol (n=22) and "HyperHAES" (n=17) effects on pyruvate concentration in "intact" brain tissue (columns - median; errors: lower - 25 percentile, upper - 75 percentile); ${ }^{*}-\mathrm{p}<0,05$ vs initial values.

Pyruvate $_{\text {les }}(\mu \mathrm{cmol} / \mathrm{l})$

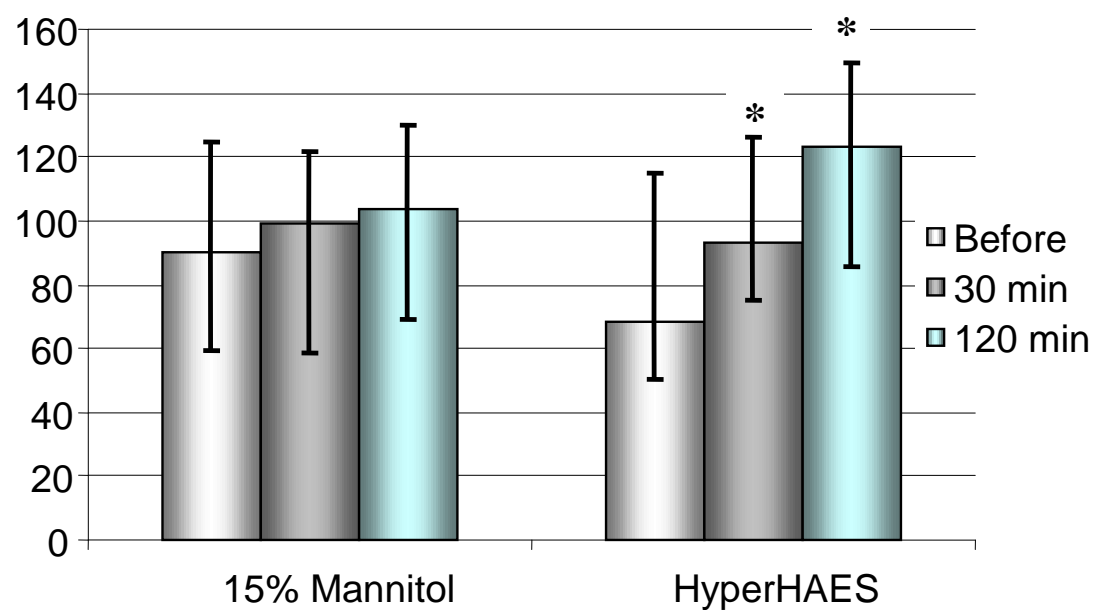

Fig. (8). 15\% Mannitol ( $\mathrm{n}=22)$ and "HyperHAES" $(\mathrm{n}=17)$ effects on pyruvate concentration in "lesioned" brain tissue (columns - median; errors: lower - 25 percentile, upper - 75 percentile); * $-\mathrm{p}<0,05$ vs initial values. 
"lesioned" areas, but was within normal values (Table 8). Lactate/pyruvate ratio increased in "intact" tissue and did not change in "lesioned" areas.

15\% Mannitol and "HyperHAES" administration did not change the glycerol concentration in "intact" and "lesioned" brain tissue.

\section{DISCUSSION}

Intracranial hypertension correction with hyperosmolar solutions is a routine practice. The results of our study confirm significant ICP reduction after 15\% Mannitol and "HyperHAES" infusion, but we observed significant difference in action duration of investigated solutions.

$15 \%$ Mannitol infusion maintained ICP below $20 \mathrm{mmHg}$ for $121 \mathrm{~min}$, "HyperHAES" - for $258 \mathrm{~min}$. This difference has vital importance in clinical conditions, because hyperosmolar agents may cause undesirable side-effects. Repeated Mannitol infusions could cause "rebound effect". Continuous administration of hyperosmolar agents increases serum osmolality and risk of renal failure [12-14].

Important data were obtained during hemodynamics parameters analysis. All patients were normovolemic before hyperosmolar solutions administration (normal values of CI, GEDVI, SVRI). Infusion of investigated solutions did not influence systemic hemodynamics, with the exception of significant increase of cardiac output at 30 minutes after "HyperHAES" infusion. Before the beginning of the study we were worried about hypervolemia that can be caused by "HyperHAES" administration. However, this was not observed.

15\% Mannitol and "HyperHAES" infusion increased cerebral perfusion pressure. However it was not associated with significant increase of brain oxygen pressure that testified to safe cerebral blood flow autoregulation in "intact" and "lesioned" brain tissue.

Intracranial hypertension was accompanied with glucose and pyruvate concentration reduction and lactate/pyruvate ratio increase in "intact" and "lesioned" brain tissue.

Brain energy demand is extremely high. Brain mass constitutes $2-3 \%$ of body mass, however, brain consumes $15 \%$ of cardiac output and $20-25 \%$ of total oxygen and glucose delivery [15]. Recent studies expanded the knowledge about cerebral metabolism in normal and ischemic conditions. Neuroglia and especially astrocytes play an important role in energy supplying of the brain [1518]. The processes of astrocytes directly contact to capillaries and form the blood-brain barrier (BBB). The major quantity of glucose goes directly into astrocytes, but not into neurons [15]. Glycolisis is the dominant process providing adenosine-three-phosphate (ATP) synthesis in astrocytes. Utilization of one glucose molecule provides formation of two pyruvate and two ATP molecules. ATP is used for maintenance of transmembrane ion gradients, ensuring functioning of ATP-dependent ion pumps (particularly $\mathrm{K}^{+}-\mathrm{Na}^{+} \mathrm{AT}$ Pase) $[15,19,20] . \mathrm{K}^{+}-\mathrm{Na}^{+}$ATPase maintains high potassium and low sodium concentration within brain cells. The major quantity of pyruvate, produced as a result of glycolisis in astrocytes, is not included in threecarbonic acids cycle and does not undergo oxidative phosphorylation, but transforms to lactate, that escapes to interstitial space and goes into neu- rons $[15,16,18]$. Currently there are a lot of evidences that lactate, but not glucose, is the main substrate for neuronal nutrition. Lactate is transformed to pyruvate, which is oxidized in respiratory chain. This process results in 18 ATP molecules production from one molecule of pyruvate. Thus, decay of one glucose molecule leads to formation of 38 ATP molecules $[15,20]$.

Sodium concentration in the brain interstitial space and in astrocytes plays the important role in cerebral metabolism. Glucose uptake from the blood is activated by increase of glutamate concentration inside the astrocytes. Glutamate uptake is associated with co-transport of 2-3 sodium ions. Thus, glutamate transport and activation of glucose uptake from the blood depends on sodium concentration in the brain interstitial space $[15,21]$.

Decrease of glucose concentration in the cerebral interstitial space increases mortality and risk of severe neurological deficit [22, 23].

Under anaerobic conditions, neuronal energy metabolism is maintained only due to glycolisis, since oxidation in respiratory chain requires oxygen. Glucose is splitted into pyruvate, with consequent transforming to lactate. Intensification of anaerobic glycolisis is accompanied with increase of lactate concentration and lactate/pyruvate ratio in brain interstitial space. But increase of lactate concentration and lactate/pyruvate ratio may occur even in normal oxygen delivery to brain tissue. This condition is defined as "metabolic crisis" and may be caused by mitochondrial dysfunction [24].

Despite similar effects on systemic hemodynamics, the influence of investigated solutions on cerebral metabolism differed significantly.

15\% Mannitol administration did not change the concentration of investigated metabolites in "intact" and "lesioned" brain tissue. Despite ICP reduction, the increase of CPP and normal brain oxygen pressure, intensity of glycolisis and oxidative phosphorilation stayed unchanged. Glucose and lactate concentrations remained significantly reduced during the study. Nevertheless, lactate concentration increased, despite the absence of significant dynamics in pyruvate concentration. It can be related to continued anaerobic glycolisis, and probably to mitochondrial dysfunction.

"HyperHAES" administration significantly increased glucose concentration in "intact" and "lesioned" brain tissue. Possibly, this effect was determined by prolong ICP reduction and increasing of CPP. However, such effects could be related to microcirculation improvement as a result of "HyperHAES" infusion [2, 25-27]. It is known, that hypertonic solutions of $\mathrm{NaCl}$ encourage the neurons membrane potential and maintain integrity of BBB [1, 28, 29]. We draw your attention to the fact, that "HyperHAES" infusion was accompanied by glycolisis activation in "intact" and "lesioned" brain tissue. However, piruvate concentration increase in the "lesioned" areas was more significant during the study. Pyruvate concentration increase was accompanied with rise of lactate concentration. Because significant increase in piruvate concentration in "lesioned" tissue was not accompanied with increase in lactate/pyruvate ratio it can be supposed that in "lesioned" areas pyruvate was oxydised in respiratory chain. In "intact" brain tissue oxidative phosphory- 
lation was impaired, since intensification of glycolisis was associated with lactate/pyruvate ratio increasing. In condition of normal oxygen supply it can be supposed the development of "metabolic crisis", caused by mitochondrial dysfunction.

We did not registrate any side-effects of investigated solutions during the whole study.

\section{CONCLUSIONS}

1. $15 \%$ Mannitol and "HyperHAES" administration is an effective method of intracranial hypertension correction. "HyperHAES" infusion results in prolong ICP reduction and cerebral perfusion pressure increase.

2. $15 \%$ Mannitol and "HyperHAES" infusion does not influence systemic hemodynamics in normovolemic patients.

3. $15 \%$ Mannitol administration does not influence cerebral oxygenation and metabolism.

4. "HyperHAES" introduction is accompanied with slight increase of cerebral oxygen tension and significant improvement of cerebral metabolism, characterized by increase of glucose and pyruvate concentration in "intact" and "lesioned" brain tissue.

5. Brain lesion, caused by intracranial hemorrhage may be accompanied by development of mitochondrial dysfunction, characterized by reduction and even enlargement of lactate/pyruvate ratio in condition of sufficient oxygen and glucose delivery to the brain.

\section{REFERENCES}

[1] Hartl R, Ghajar J, Hochleuthner H, Mauritz W. Treatment of refractory intracranial hypertension in severe traumatic brain injury with repetitive hypertonic/hyperoncotic infusions. Zentralbl Chir 1997; 122(3): 181-5.

[2] Kempski O, Obert C, Mainka T, Heimann A, Strecker U. "Small Volume Resuscitation" as treatment of cerebral blood flow disturbances and increased ICP in trauma and ischemia. Acta Neurochir Suppl 1996; 66: 114-7.

[3] Thomale U, Griebenow M, Kroppenstedt SN, Unterberg AW, Stover JF. Small volume resuscitation with HyperHaes improves pericontusional perfusion and reduces lesion volume following controlled cortical impact injury in rats. J Neurotrauma 2004; 21(12): 1737-46.

[4] Bhardwaj A, Ulatowski JA. Cerebral edema: hypertonic saline solutions. Curr Treat Options Neurol 1999; 1(3): 179-88.

[5] Harukuni I, Kirsch JR, Bhardwaj A. Cerebral resuscitation: role of osmotherapy. J Anesth 2002; 16(3): 229-37.

[6] Wakai A, Roberts IG, Schierhout G. Mannitol for acute traumatic brain injury. Cochrane Database Syst Rev (Issue 1) 2007: Art. No.: CD001049.

[7] Vialet R, Albanèse J, Thomachot L, et al. Isovolume hypertonic solutes (sodium chloride or mannitol) in the treatment of refractory posttraumatic intracranial hypertension: $2 \mathrm{~mL} / \mathrm{kg} 7.5 \%$ saline is more effective than $2 \mathrm{~mL} / \mathrm{kg} 20 \%$ mannitol. Crit Care Med 2003; 31(6):1683-7.

[8] Battison C, Andrews PJ, Graham C, Petty T. Randomized control trial on the effect of a $20 \%$ mannitol solution and a $7.5 \%$ saline $/ 6 \%$ dextran solution on increased intracranial pressure after brain injury. Crit Care Med 2005; 33(1): 196-202.
[9] Al-Rawi PG, Zygun D, Tseng MY, Hutchinson PJ, Matta BF, Kirkpatrick PJ. Cerebral blood flow augmentation in patients with severe subarachnoid haemorrhage. Acta Neurochir Suppl 2005; 95:123-7.

[10] Sakowitz OW, Stover JF, Sarrafzadeh AS, Unterberg AW, Kiening KL. Effects of mannitol bolus administration on intracranial pressure, cerebral extracellular metabolites, and tissue oxygenation in severely head-injured patients. J Trauma 2007; 62(2): 292-8.

[11] Reinstrup $\mathrm{P}$, Ståhl N, Mellergård P, Uski T, Ungerstedt U, Nordström CH. Intracerebral microdialysis in clinical practice: baseline values for chemical markers during wakefulness, anesthesia, and neurosurgery. Neurosurgery 2000; 47(3): 701-9.

[12] Garcia-Sola R, Pulido P, Capilla P. The immediate and long-term effects of mannitol and glycerol: a comparative experimental study. Acta Neurochir (Wien) 1991; 109(3-4):114-21.

[13] Node Y, Yajima K, Nakazawa S. Rebound phenomenon of mannitol and glycerol: clinical studies. No To Shinkei 1983; 35(12):1241-6.

[14] Prough DS, Whitley JM, Taylor CL, Deal DD, DeWitt DS. Rebound intracranial hypertension in dogs after resuscitation with hypertonic solutions from hemorrhagic shock accompanied by an intracranial mass lesion. J Neurosurg Anesthesiol 1999; 11(2): 102-11.

[15] Pellerin L. How astrocytes feed hungry neurons. Mol Neurobiol 2005; 32(1): 59-72.

[16] Bonvento G, Herard A, Voutsinos-Porche B. The astrocyte-neuron lactate shuttle: a debated but still valuable hypothesis for brain imaging. J Cereb Blood Flow Metab 2005; 25(10): 1394-9.

[17] Loaiza A, Porras O, Barros L. Glutamate triggers rapid glucose transport stimulation in astrocytes as evidenced by real-time confocal microscopy. J Neurosci 2003; 23(19): 7337-42.

[18] Magistretti P, Pellerin L. Astrocytes couple synaptic activity to glucose utilization in the brain. News Physiol Sci 1999; 14: 177 - 82.

[19] Bergsneider M, Hovda DA, Shalmon E, et al. Cerebral hyperglycolysis following severe traumatic brain injury in humans: a positron emission tomography study. J Neurosurg 1997; 86(2): 241-51.

[20] Kann O, Kova'cs R. Mitochondria and neuronal activity. Am J Physiol Cell Physiol 2007; 292(2): C641-57.

[21] Pellerin L, Magistretti P. Glutamate uptake into astrocytes stimulates aerobic glycolysis: a mechanism coupling neuronal activity to glucose utilization. Proc Natl Acad Sci USA 1994; 91(22): 10625-9.

[22] Parkin M, Hopwood S, Jones DA, et al. Dynamic changes in brain glucose and lactate in pericontusional areas of the human cerebral cortex, monitored with rapid sampling on-line microdialysis: relationship with depolarisation-like events. J Cereb Blood Flow Metab 2005; 25: 402-13.

[23] Vespa P, McArthur D, O'Phelan K, et al. Persistently low extracellular glucose correlates with poor outcome 6 months after human traumatic brain injury despite a lack of increased lactate: a microdialysis study. J Cereb Blood Flow Metab 2003; 23(7): 865-77.

[24] Vespa P, Bergsneider M, Hattori N, et al. Metabolic crisis without brain ischemia is common after traumatic brain injury: a combined microdialysis and positron emission tomography study. J Cereb Blood Flow Metab 2005; 25(6): 763-74.

[25] Berger S, Schürer L, Härtl R, Messmer K, Baethmann A. Reduction of post-traumatic intracranial hypertension by hypertonic/hyperoncotic saline/dextran and hypertonic mannitol. Neurosurgery 1995; 37(1): 98-107.

[26] Hannemann L, Reinhart K, Korell R, Spies C, Bredle DL. Hypertonic saline in stabilized hyperdynamic sepsis. Shock 1996; 5(2): 130-4.

[27] Tseng M, Al-Rawi PG, Czosnyka M, et al. Enhancement of cerebral blood flow using systemic hypertonic saline therapy improves outcome in patients with poor-grade spontaneous subarachnoid hemorrhage. J Neurosurg 2007; 107(2): 274-82.

[28] Cross JS, Gruber DP, Burchard KW, Singh AK, Moran JM, Gann DS. Hypertonic saline fluid therapy following surgery: a prospective study. J Trauma 1989; 29(6): 817-25.

[29] Hartl R, Ghajar J, Hochleuthner H, Mauritz W. Hypertonic/hyperoncotic saline reliably reduces ICP in severely headinjured patients with intracranial hypertension. Acta Neurochir Suppl 1997; 70: 126-9. 